\title{
IMPLEMENTACIÓN DEL MAPA DE GEOPEDOLOGÍA COMO BASE PARA LA DELIMITACIÓN DE UNIDADES DE ECOSISTEMAS A NIVEL NACIONAL EN COLOMBIA
}

\section{IMPLEMENTATION OF THE MAP OF GEOPEDOLOGY AS A BASIS FOR DELIMITING ECOSYSTEM UNITS A NATIONAL LEVEL IN COLOMBIA}

\author{
Carlos Enrique Castro Méndez ${ }^{1}$, Yolima del Carmen Agualimpia Dualiby ${ }^{2}$
}

\begin{abstract}
${ }^{1}$ Agrólogo, Geógrafo, Especialista en Cuencas Hidrográficas, Magíster Geografía. Investigador Progasp, Calle 127 bis 88-07 interior 7 casa 26, Bogotá, Colombia, e-mail: cecastro77@gmail.com; ${ }^{2}$ Ingeniera Civil, Magíster Ingeniería Civil, Doctora Ciencias Técnicas, Docente. Universidad Distrital Francisco José de Caldas e Investigador Progasp, Calle 127 bis 88-07 interior 7 casa 26, Bogotá, Colombia, e-mail: yagualimpiadualiby@gmail.com
\end{abstract}

Rev. U.D.C.A Act. \& Div. Cient. 20(1): 175-185, Enero-Junio, 2017

\section{RESUMEN}

Geopedología corresponde a la integración entre geomorfología y pedología; es el concepto que se aplica en Colombia, para interpretar la información de los suelos y, a través de ella, generar cartografía temática, que pueda ser representada en diferentes escalas. Una aplicación reciente de la geopedología es la definición del medio pedológico como sustrato, que soporta ecosistemas terrestres y que hace parte del ambiente, debido a que cumple la función de permitir los flujos de energía entre coberturas vegetales y seres vivos. En este artículo, se realiza una reflexión sobre el papel que, históricamente, ha tenido la información de los suelos en el contexto colombiano y su aporte a las ciencias del ambiente; se muestra un desarrollo histórico, que permite analizar cómo se ha integrado la información de suelos realizada en el Instituto Geográfico Agustín Codazzi (IGAC), por más de setenta años y cómo el mejoramiento continuo ha permitido el acercamiento teórico a la delimitación de ecosistemas para dos escalas: la nacional y la general. La ciencia del suelo ha tenido poco reconocimiento en la elaboración de informes recientes relacionados con el ambiente, por ello, es importante que se muestren conceptos y trabajos realizados por expertos en suelos y se divulgue la labor de quienes la desarrollan, así como los resultados obtenidos en la investigación realizada por Progasp, relacionado con el tema de escalas y de representación de variables, que permiten mostrar la realidad ambiental y avance en los procesos que, actualmente, se llevan a cabo en Colombia.

\section{SUMMARY}

Geopedology corresponds to the integration between geomorphology and soil science and this concept is applied in Colombia to interpret the information of soil for generating thematic maps that can be displayed on different scales; a recent application of the geopedology is the definition of pedological zone as the substrate that supports terrestrial ecosystems and is part of the environment because it serves to allow the energy flows between mulches and living things. This article presents a reflection on the role that historically has had the soils information in the Colombian context and its contribution to environmental science; a historical development is done about soil information to analyze its integration in the Instituto Geográfico Agustín Codazzi (IGAC) for over seventy years and how the continuous improvement has allowed the theoretical approach and the delimitation of ecosystems are close to each other to two scales: national and overall. Soil science has had little recognition in the development of recent reports related to the environment, so it is important that concepts and work done by experts in soils are shown; it is equally important that result obtained in the investigation performed by the research group Progasp, related to the topic of scales and representation of variables which allow display environmental reality and the processes that are advancing in Colombia.

Key words: Geomorphology, pedology, ecosystem and soil genesis.

Palabras clave: Geomorfología, pedología, ecosistemas y génesis del suelo. 


\section{INTRODUCCIÓN}

Conocer los recursos naturales con que cuenta Colombia requiere de un análisis integral de elementos, que han servido para la identificación y la delimitación del sistema ambiental. El enfoque ambiental, basado en ecosistemas, como se concibe en la actualidad, no tiene en cuenta en sus informes técnicos al suelo, como generador de servicios. La normatividad utilizada en el manejo del suelo aplica el enfoque productivo y por tal motivo, esta información no es visible en los informes que se han realizado del ambiente. En la Política para la Gestión Integral Ambiental del Suelo generada por el Ministerio de Ambiente y Desarrollo Sostenible (2013), se afirma que ante la ausencia de una definición unificada no es posible aplicar una reglamentación ajustada a los propósitos del gobierno, debido a que varias organizaciones gubernamentales administran territorio. Las normas que producen estas entidades están desarticuladas y esto dificulta la gestión ambiental del suelo; no obstante, la información de suelos ha sido un elemento utilizado por el Instituto de Hidrología, Meteorología y Estudios Ambientales (IDEAM, 2001), en los informes de estado de los recursos naturales y en la delimitación de los ecosistemas terrestres; por ello, es necesario conocer los aportes de la ciencia del suelo y los primeros enfoques ambientales desarrollados, a partir de esa información en Colombia.

La ciencia del suelo es reciente. El primer registro de estudio agrológico aparece en México, en el sistema nacional de riego Coahuila (Rico, 1931). En 1940, se abre la oficina de Catastro Rural en el Instituto Geográfico Militar de Colombia y se realizan estudios de caracterización de suelos, para cumplir con las necesidades de información cartográfica (IGAC, 2016). En los años cincuenta, se ejecutan trabajos para adecuar tierras con drenaje natural pobre, caso de valle del Sibundoy (IGAC, 1955) e instalar el distrito de riego en el río Coello, en el departamento de Tolima (IGAC, 1955), así como en zonas del altiplano Tunja- Sogamoso (IGM, 1957).

Los estudios de suelos realizados en los años cincuenta correspondían a zonas planas, que cumplían con el requerimiento de ser arables; los suelos alóctonos o formados por materiales transportados, se caracterizaban con texturas y colores. Posteriormente, los estudios se extendieron a zonas con relieve, en los cuales, se incluyó la pendiente y otras variables, derivadas de procesos que suceden en relieves fuertes, tales como la pedregosidad, el afloramiento de rocas y la erosión. Los primeros estudios incluían la distribución de variables de suelos que se aplicaban al catastro rural, mediante el índice de productividad de las tierras (Cortés, 1974).

En los años sesenta, el auge de levantamiento de suelos en regiones planas y con fines agrícolas, continuaría en el valle del río Zulia, en Norte de Santander (IGAC, 1964) y estudios, con fines catastrales, en los municipios de San Zenón y San Sebastián, en Magdalena (IGAC, 1968).

Los estudios de suelos han sido realizados durante sesenta años para definir el uso y manejo, orientado a la productividad agrícola. Con la promoción de agrólogos preparados para orientar el uso de suelos agrícolas, comienza la investigación aplicada en la sabana de Bogotá (Malagón, 1966; 1968; 1969); se abordaron temas de mineralogía (Malagón, 1975) y física de suelos (Montenegro y Malagón, 1990), importantes en el desarrollo de la ciencia del suelo en Colombia. Entre los años sesenta y setenta, se oficializa el uso de la taxonomía de suelos del Departamento de Agricultura de los Estados Unidos (USDA) y se estructura la correspondiente leyenda.

Los primeros estudios de suelos realizados en Colombia fueron un claro desempeño de profesionales en química, quienes alternaban sus evaluaciones con aportes de agrónomos que, en condiciones de invernadero, analizaban la respuesta de los cultivos a aplicaciones de nitrógeno, fósforo y potasio; estos trabajos dieron origen a la tabla de fertilidad, que se utiliza en la actualidad (IGAC, 2007).

La pedología avanzó en el estudio de las propiedades del suelo, dando elementos para determinar el uso y el manejo agrícola. Las propiedades ándicas de los suelos derivados de cenizas volcánicas, se estudiaron, incluso, antes de que se incluyera el orden taxonómico en la clave (IGAC, 1981), se adoptó el esquema jerárquico de geomorfológica propuesto por Zinck (1987) y se estudió la relación suelo paisaje, en la que se contemplaban características fisicoquímicas (IGAC, 1995).

Entre los años ochenta y los noventa, se introdujeron las clases agrológicas con ajustes al medio colombiano, para pasar de estudios de suelos de tipo descriptivo a los aplicados al uso agrícola y la conservación o recuperación (IGAC, 1986), independizando el proceso catastral, del cual, se definía el valor potencial del suelo para áreas homogéneas de tierra, para el catastro (IGAC, 1981).

Desde los años noventa hasta el 2015, los esfuerzos del IGAC se centraron en la integración de estudios sectoriales hasta el nivel departamental. La información, a nivel nacional, se presentó en tres versiones: Mapa de Suelos de Colombia escala 1:1.500.000 (IGAC, 1982a), Mapa de Suelos de Colombia escala 1:500.000 y Mapa de Suelos de Colombia escala 1:100.000 (IGAC, 2015). Durante seis décadas, se realizaron levantamientos de suelos en muchos sectores del territorio colombiano. La consulta e integración de alrededor de quinientas memorias técnicas junto con la estructuración de esta información, bajo la mirada nacional, permitieron generar datos del suelo, que sirvieron para mostrar el estado de este recurso en Colombia (IGAC, 2016). 
Los primeros trabajos realizados hacia un enfoque ambiental, se dieron a través del mapa de geopedología, considerado un producto de transición, aplicado a caracterizar el sustrato de los ecosistemas. El concepto ecosistema, acuñado por los botánicos Clapham y Tansley, en 1930, tiene su aplicación a diferentes escalas (Raffaelli, 2010); cada país representa, de manera diferente, la interacción entre organismos vivos (biocenosis) y el medio físico (biotopo), el cual, es denominado hábitat. Al interior de los ecosistemas, se consideran flujos internos de materia y energía y en sus límites; Clements (1905) lo denominó ecotono.

El ciclo de la vida dentro del hábitat fue identificado por Darwin (2011), quien demostró que el mantillo del suelo es producto de nacimiento y muerte de las lombrices. El flujo de energía en la formación de los suelos fue estudiado también por Volobuev (1964), quien utilizó el clima ambiental como variable derivada del balance de radicación neta de la superficie terrestre, determinada a partir de siete termosecuencias y once hidrosecuencias; además, en el suelo, se realizan cuatro procesos ecológicos que dinamizan el sistema: 1) el agua (White, 2006); 2) los nutrientes (Bohlen \& House, 2009); 3) los flujos energéticos (Begon et al. 1996) y 4) la dinámica de las comunidades o sucesiones vegetales (Sarmiento, 1982). Los suelos, se cargan de energía mediante el ciclado de nutrientes, los aportes de sedimentos o la acumulación de humus y consumen energía durante la evolución, al pasar por estados de mineralización, de liberación y de transferencia de nutrientes (Zapata, 2006). Las coberturas vegetales participan en la evolución del suelos, así lo entendieron los ecólogos Aber \& Melillo (2001), quienes relacionaron los órdenes taxonómicos de suelos con los biomas.

De acuerdo con los anteriores desarrollos conceptuales, el suelo se considera elemento determinante en la delimitación de ecosistemas terrestres; a través del suelo, se puede representar el registro de ecosistemas originales y los procesos que han sucedido en el medio ambiente. El suelo almacena carbono orgánico, como proceso de combustión y el uso inadecuado del mismo puede generar cambios en el clima y en el hábitat de especies; así fue interpretado en la cartografía de ecosistemas a la escala 1:100.000 (MINAMBIENTE et al. 2015).

La razón de la presente investigación es resaltar la integración de suelos realizada en Colombia y la importancia de estos en los temas ambientales. Se parte del supuesto de que el suelo es sustrato de los sistemas ambientales y, a partir del conocimiento de éste, es posible delimitar los ecosistemas terrestres.

\section{MATERIALES Y MÉTODOS}

- Estudios de suelos y planchas temáticas

- Manuales de procedimiento aplicados en el levantamiento de suelos

- Literatura sobre temas ambientales

- Mapas y memorias técnicas de geopedología a escala $1: 500.000$ y $1: 100.000$

- Shapefile de sectores de la altillanura y depresión intramontana

El estudio consistió en revisar documentos para conocer el proceso aplicado en la integración de información de suelos y las bases científicas de expertos, para responder las necesidades de información con enfoque ambiental, a dos escalas de representación.

Se hizo un análisis retrospectivo de la cartografía de suelos, a través de entrevistas a expertos, quienes trabajaron la temática en Colombia; se complementó la información con registros bibliográficos y manuales utilizados en la elaboración, análisis y diseño de los mapas de suelos de Colombia. El método consistió en analizar los procedimientos aplicados por científicos del suelo e identificar los desarrollos conceptuales aplicados a cada escala. Los pasos generales fueron: 1) Revisión de informes técnicos de suelos, investigaciones y manuales de procedimiento de levantamientos de suelos, en el contexto nacional; 2) Análisis y contraste entre informes y cartografía temática en otros países; 3) Identificación de conceptos y de variables aplicadas en Colombia, a cada escala en el tema de geopedología y 4) análisis del proceso utilizado en la integración de información de suelos y escogencia de las variables aplicadas en Colombia, durante la generación del mapa de geopedología.

\section{RESULTADOS Y DISCUSIÓN}

La primera versión del mapa de suelos de Colombia la realizó el IGAC, a escala 1:1.500.000 (IGAC, 1982a). Para la conformación de este producto, se utilizó el mapa de zonas de vida, publicado a escala 1:500.000, por la misma institución, en 1977, los estudios generales y regionales de la época, y las cartas geológicas del Instituto Colombiano de Geología y Minería (INGEOMINAS), de 1976.

En el mapa de suelos, versión de 1982, se aplicó un enfoque pedológico, en el cual, el material parental era trasformado por el clima y cuyo efecto se interpretaba en el tipo de cobertura vegetal. Las unidades cartográficas agrupadas por paisajes geomorfológicos representa la morfogénesis dominante, asociada al origen del suelo. 
El proceso utilizado para generar y estructurar 1.041 hojas cartográficas de suelos y plasmar la distribución de los suelos en 26 planchas 1:500.000, en el 2003, requirió de dos elementos base, para homogenizar los criterios de conformación de unidades: la zonificación climática y la definición de materiales parentales, provenientes de los mapas de suelos y de geología.

Para definir las unidades climáticas, se digitalizó el mapa de zonas de vida de Colombia y se hizo la reclasificación a pisos térmicos y condiciones de humedad; esta información, se cruzó con el nuevo mapa geomorfológico y se agregó el correspondiente suelo dominante. El mapa de suelos de Colombia escala 1:500.000 de contaba con 197 unidades cartográficas.

La construcción de la información de suelos a escala 1:100.000, en el 2015, requirió la integración de nuevos estudios departamentales, por el grupo de expertos. La cartografía temática fue estructurada mediante consulta de 182 documentos técnicos, producidos durante los 70 años de historia del IGAC. También, se utilizaron datos reportados en 109 estudios agrológicos especiales, que incluían los temas de cobertura vegetal, investigaciones aplicadas a suelos y temas afines. El mapa de suelos contiene 1.475 unidades cartográficas, cuyo contenido pedológico se agrupa en 11 órdenes de suelos y 59 subórdenes taxonómicos en la cartografía temática, sin incluir los gelisoles (IGAC, 2015).

Para la configuración de las 1475 unidades cartográficas, se utilizaron 3.238 perfiles, provenientes de los estudios generales de suelos. La mayoría de las descripciones de suelos se situaban en los departamentos de la región andina y caribe.

El mapa de geopedología, realizado por el IGAC, en el 2007, para el proyecto ecosistemas continentales, costeros y marinos de Colombia fue definido, de acuerdo con dos componentes básicos: la cartografía de suelos armonizada a escala 1:500.000 y el paisaje geomorfológico integrado; estos productos fueron mejorados en el nivel superior e inferior. La zonificación climática fue la capa de información más general; le siguió el ambiente morfogenético que, según Zinck (1988), corresponde a acciones que originaron los relieves actuales, enmarcados en paisajes geomorfológicos y, finalmente, en la modelación del relieve. Esta delimitación fue trabajada bajo bases conceptuales, que permitieron la integración de tipos de relieve, según el proceso general, determinado en la formación de estos.

Confrontación con otras experiencias en el mundo. Birkeland (1999) concluyó que si se evaluaba la evolución del relieve al igual que la edad y la estabilidad de los procesos junto con el clima del pasado, se tenían elementos adecuados para delimitar los suelos. Gerrard (1992) propuso evaluar las relaciones genéticas del relieve para identificar los suelos. El enfoque geopedológico aplicado por Zinck (1988) incluyó cuatro aspectos complementarios: morfometría, morfogénesis, morfocronología y morfodinámica.

La integración de información de suelos y geomorfología propuesta por Gerrard (1981) y Zinck (1988) contribuyó a mejorar los levantamientos de suelos; le siguió el análisis fisiográfico, como enfoque práctico de la geomorfología climática, desarrollada, años atrás, por Peltier (1950), Stoddart (1968) y Tricart (1977). El avance de esta metodología y el concepto que relaciona el suelo con el paisaje, se presentan en el libro de Geopedology, de Zinck (2013), en el cual, trató la escala de representación de las geoformas y suelos (Zinck, 1987); el tema adquirió mayor relevancia y actualidad en la publicación Geopedology An Integration of Geomorphology and Pedology for Soil and Landscape Studies (Zinck et al. 2016).

La geopedología aplicada por Wild (1993), a temas ambientales, consideraba al suelo como sustrato de los ecosistemas terrestres. En Brasil, se realizaron varias versiones del mapa integrado de geopedología, entre 1981 y 1993, donde las representaciones cartográficas se modificaron, de acuerdo con los nuevos criterios y atributos aportados por el Departamento de Agricultura de los Estados Unidos, por sus siglas en inglés USDA (1992) y la Organización de las Naciones Unidas para la alimentación y la agricultura, por sus siglas en inglés FAO (1993a, 1993b); los temas ambientales se representaron en Brasil, a la escala 1:5.000.000, debido a lo extenso del territorio (Teixeira \& Baptista, 2000).

La geomorfología y la pedología fueron consideradas por Fitzpatrick (1971), Hugget (2003), Anderson \& Anderson (2010) y Gutierrez (2013), como ciencias físicas independientes; sin embargo, las investigaciones realizadas por Zinck (1970; 1974; 1977; 1980) permitieron integrar la relación suelo-paisaje para su aplicación en temas, como la biodiversidad (lbáñez y Pérez, 2015), relación entre suelos y formas eólicas y fluviales (Abdelfattah, 2013) y permitió la generación de nuevos enfoques al delimitar geoformas, según la escala requerida (Abdelfattah \& Pain, 2012). Con relación a la escala de representación, en Colombia se avanzó en el tema de uso potencial de la tierra, según el nivel de exactitud de la escala: a nivel general, los usos principales y, a nivel detallado, los usos específicos (IGAC, 2007).

Toomanian (2013) estudió la distribución de formas del relieve y suelos en diferentes regiones de Irán y encontró patrones similares, en cuanto a la biodiversidad; sugirió la regularidad entre funciones que relacionan estructuras ecológicas, biológicas y bióticas, por tanto, argumentaron la 
utilidad del enfoque pedológico, en la delimitación de los ecosistemas.

La leyenda del mapa de geopedología de Colombia muestra avances conceptuales en la integración de conocimientos, basados en la relación suelo-paisaje. En primera instancia, la escala de trabajo y la escala final permitieron definir la representatividad de las variables. En el mapa de suelos (IGAC, 1982a) fue necesario readecuar la información cartográfica temática, de escala 1:500.000 a 1:1.500.000, para justificar el grado de incertidumbre; debido a la escasa información que se tenía en la década de los ochenta, se agruparon paisajes y se asignaron cualidades que representaban el origen de los suelos, según sea el proceso fluvial, erosional o estructural, entre otros. Las características de los suelos fueron asociadas a relieves, según categorías de pendiente, e drenaje natural y de clima atmosférico.

La leyenda del mapa de geopedología escala 1:500.000, se estructuró con base en la información del paisaje, ambiente morfogenético, pendiente, drenaje y órdenes de suelos; el símbolo representaba siete paisajes: montaña, piedemonte, lomerio, altiplanicie, superficies de aplanamiento, planicie y valle; los ambientes morfogenéticos, definidos a esta escala nacional, fueron siete: estructural-erosional, glaciárico, fluvio-gravitacional, coluvio-aluvial, aluvial, residual y eólica. Las pendientes asociadas a las formas del relieve fueron siete: menores o mayores del $7 \%$, menores o mayores al $50 \%$; menores o mayores al $25 \%$ y una categoría indeterminada; los drenajes, se agruparon en dos clases: imperfecto a excesivo y pobre a muy pobre. Cada ambiente morfogenético fue relacionado con los once órdenes taxonómicos dominantes, reportados en estudios anteriores al 2002. Al final, se obtuvieron 30 unidades geopedológicas a escala 1:500.000, que serían la base de los ecosistemas definidos, según el clima, las coberturas vegetales, los tipos de suelos y el relieve.

La estructuración del mapa de geopedología de Colombia a escala 1:100.000, se realizó a partir del correspondiente mapa de suelos (IGAC, 2015). Al ser un estudio de mayor resolución que el del 2003, se obtuvo un análisis detallado de las unidades cartográficas, bajo el criterio del marco evolutivo del suelo, según el paisaje, clima, tipo de relieve, material de origen y drenaje.

Mientras en el primer mapa de geopedología (escala 1:500.000), se aplicó un método deductivo, basado en suelos comunes, en una geoforma; en la nueva versión (escala $1: 100.000$ ), se utilizó un método inductivo, en el cual, se le dio mayor relevancia a los procesos pedogéneticos que representan los suelos, identificados en una geoforma específica.
Durante el análisis de la relación suelo-paisaje, los expertos adoptaron, como criterio, la condición básica para el ambiente de formación; aplicaron, como marco general, el ambiente morfogenético del paisaje y luego el análisis de suelo, como resultado de la condición regional, que incide en la pedogénesis, determinada por el clima edáfico y el material de origen del mismo. La vegetación, en este sentido, no fue considerada como factor determinante en la formación, sino como indicador de la condición actual, ya que en algunas ocasiones la morfología del suelo no reflejaba el clima edáfico y, en su defecto, sí las condiciones climáticas pasadas.

La cantidad de unidades cartográficas de suelos del mapa nacional de suelos (IGAC, 2015) no fue impedimento para la delimitación de ecosistemas terrestres. Los pedólogos crearon categorías homogéneas de paisaje y suelo, que les permitió generar el mapa de geopedología, a partir de la relación entre la geomorfología y los suelos. Los expertos analizaron el grado y la intensidad de los procesos pedogenéticos, de acuerdo con el nivel taxonómico de mayor jerarquía que el orden y escogieron el subgrupo.

A escalas de mayor detalle, se pueden distinguir los procesos químicos que tienen lugar en los suelos y, especialmente, se pueden identificar los flujos energéticos, mediante el análisis del tipo de humus y cuantificación de los elementos liberados durante los procesos pedogenéticos (Sarmiento, 1982). Con base en lo anterior, los especialistas de suelos colombianos examinaron 1.475 unidades cartográficas de suelos, de acuerdo con una o más características combinadas del paisaje, agrupadas por tipos de relieve y confrontadas con los procesos de formación de suelos. En total generaron 254 unidades cartográficas de geopedología, para todo el país.

Para mostrar la aplicación de conceptos en la representación de la geopedología, se tomaron, como estudio de caso, dos regiones de interés actual, una muestra de la altillanura y una zona productiva de paisaje valle. El procedimiento consistió en identificar las condiciones, bajo las cuales, se formaron y agruparon los suelos, según la intensidad y la característica del paisaje. Se utilizó información secundaria en escalas 1:25.000 (IGAC, 1989) y 1:100:000 (IGAC, 1982b), ajustada con fotointerpretación de paisajes y relieves para representar los conceptos, de acuerdo con la distribución espacial a la escala general (1:100.000), contrastada con la escala nacional (1:500.000).

El elemento formador cartografiado en el tema de geopedología escala 1:100.000 (IGAC, 2013), representa el estado genético del suelo interpretado por expertos, para la escala general; consistió en agrupar suelos de diferentes órdenes taxonómicos, cuya característica común fue el régimen de 
humedad, los procesos de humificación, lixiviado de nutrientes, características relacionadas con el tipo de arcilla, acumulación de arenas provenientes de procesos de despositación aluvial o suelos residuales, salinidad, sodicidad, procesos de andolización, oxidación, intemperización y aridez, entre otras.
Al comparar el mapa de geopedología realizado mediante los procedimientos a las zonas de altillanura y depresión intramontana con el mapa de ecosistemas, producido por el MINAMBIENTE et al. (2015) escala 1:100.000, se encontró que hay alta correspondencia (Tabla 1$)$.

Tabla 1. Correspondencia entre unidad geopedológica y ecosistemas.

\begin{tabular}{|c|c|c|c|}
\hline Unidad geopedológica & Zona geográfica & Características & Ecosistema \\
\hline 1 & Depresión intramontana & $\begin{array}{l}\text { Relieve plano-cóncavo y } \\
\text { drenaje natural restringido }\end{array}$ & $\begin{array}{l}\text { Bosque de galería } \\
\text { basal húmedo }\end{array}$ \\
\hline 2 & Altillanura & $\begin{array}{l}\text { Relieve plano y cóncavo con } \\
\text { acumulación con alto \%M.O }\end{array}$ & $\begin{array}{l}\text { Bosques de galería } \\
\text { inundable basal }\end{array}$ \\
\hline 3 & Altillanura & $\begin{array}{l}\text { Relieve plano-convexo con } \\
\text { depósitos de arenas }\end{array}$ & Sabanas inundables \\
\hline 6 & Altillanura & $\begin{array}{l}\text { Paisajes residuales sometidos } \\
\text { a lavados de nutrientes }\end{array}$ & Sabanas estacionales \\
\hline 7 & Altillanura & $\begin{array}{c}\text { Geoformas antiguas con } \\
\text { materiales muy meteorizados }\end{array}$ & Agroecosistema ganadero \\
\hline 9 & Depresión intramontana & $\begin{array}{c}\text { Paisajes relativamente altos o levanta- } \\
\text { dos por procesos orogenéticos, con } \\
\text { materiales permeables }\end{array}$ & $\begin{array}{c}\text { Agroecosistemas } \\
\text { mosáico de cultivo y pastos }\end{array}$ \\
\hline 13 & Depresión intramontana & $\begin{array}{c}\text { Geoformas por sedimentación reciente } \\
\text { y pedogénesis moderada }\end{array}$ & Río de aguas claras \\
\hline
\end{tabular}

Esto demuestra, el aporte de la información de suelos y la implementación del mapa de geopedología en la delimitación de los ecosistemas, a la escala 1:100.000.

La figura 1 - parte A, ilustra la distribución de unidades geopedológicas en la altillanura colombiana, en el sector de los ríos Tillava e Iteviare, en Puerto, Gaitán (Meta). Al aplicar el procedimiento definido para reinterpretar la información de suelos, se distinguieron cuatro unidades geopedológicas, así: la unidad 2, definida por acumulación de materia orgánica, con bajo proceso de humificación; la unidad 3, definida por acumulación de arenas eólicas y depósitos aluviales, con suelos de poco desarrollo; la unidad 6 , constituida por paisajes de altillanura, con materiales residuales y suelos en estado avanzado de pedogénesis y la unidad 7, definida por paisajes residuales de altillanura, con meteorización muy alta.

La figura 1 - parte $B$, muestra la distribución de unidades geopedológicas halladas por aplicación del procedimiento a un estudio reciente, en un pequeño sector de los municipios de Puerto Santander y Cúcuta (Norte de Santander), entre los ríos Zulia y Pamplonita. En la zona predomina la unidad 1 , correspondiente al paisaje de valle y cuya condición es áquica y con proceso mínimo de oxidación; la unidad 9, que contiene paisajes deposicionales, localmente altos, que generan condición de oxidación con lavado de bases y alta permeabilidad, mientras la unidad 13 , del paisaje valle, contiene sedimentos recientes y cuyos suelos tienen pedogénesis moderada y mayores posibilidades de uso agrícola.

La representación de la geopedología a escala nacional 1:500.000 (Figura 1A), corresponde a dos unidades geopedológicas nacionales: la altillanura estructural, agrupación de los códigos 6 y 7 y el lomerío estructural erosional, producto de la agrupación de códigos 2 y 3 . En la figura 1B, se distingue una unidad geopedólogica nacional, la cual, corresponde a la agrupación de los códigos 1, 3 y 9, del paisaje de valle intramontano deposicional, esto permite demostrar que hay un orden de representación de la geopedología y un avance conceptual aplicado a dos escalas diferentes, en el cual, se incluyen variables representativas del suelo, durante la delimitación de los ecosistemas terrestres, a escalas grandes y a escalas pequeñas variables de paisaje. 

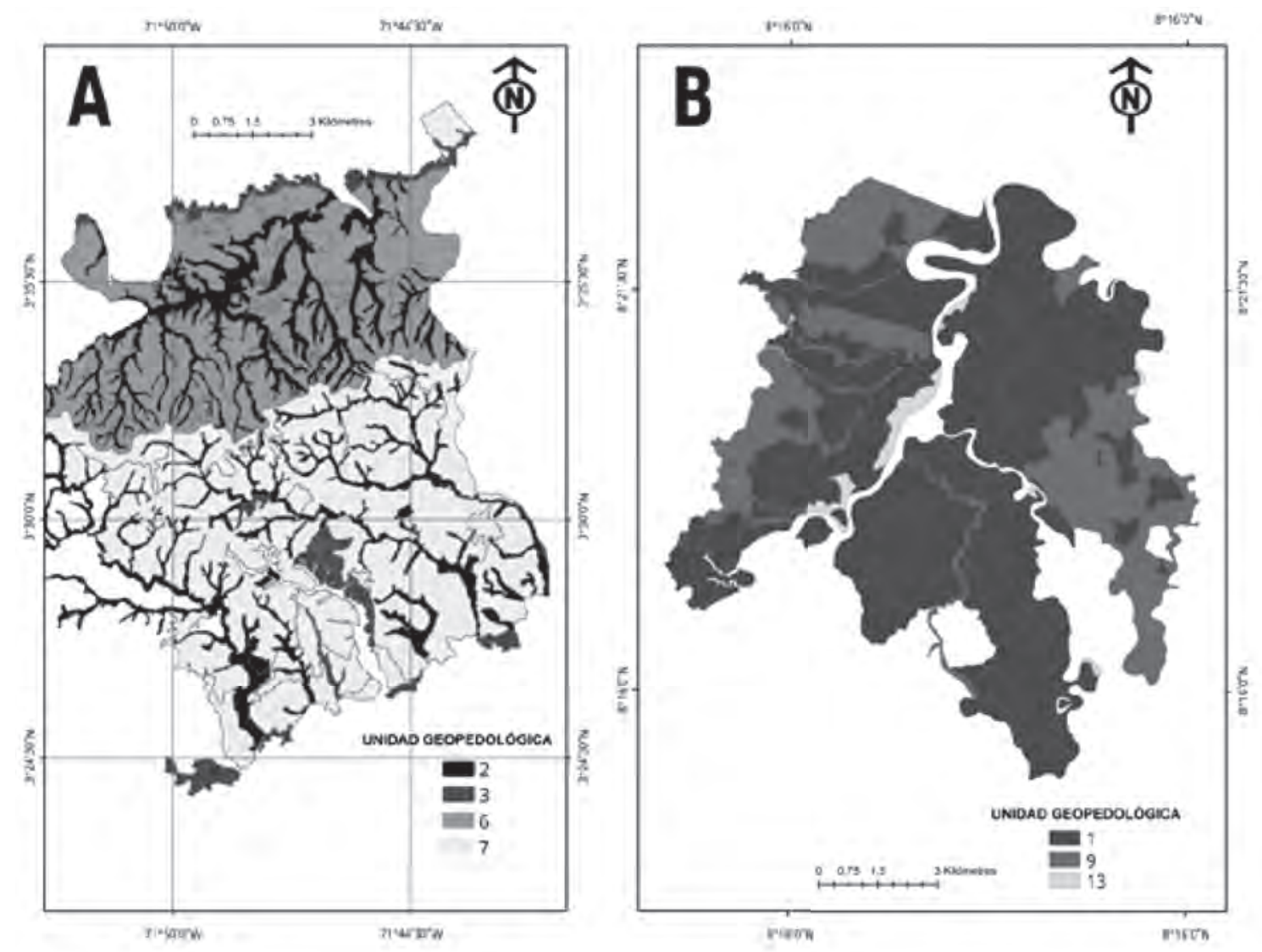

Figura 1. Distribución de unidades geopedológicas en sector de la altillanura, Parte A y en valle intramontano, Parte B.

\section{CONCLUSIONES}

La realización de estudios de suelos, a escalas de mayor detalle, permite mejorar el conocimiento del recurso con la aplicación de nuevas herramientas, actualización de manuales de reconocimiento y conceptualización; permite, además, identificar procesos pedológicos ocultos a la escala nacional y mejorar la comprensión, para delimitar los ecosistemas, en un país de alta biodiversidad de especies.

Los especialistas de suelos aplicaron aspectos relacionados con los procesos de configuración de paisajes y ambientes de formación de los suelos, al conformar la cartografía de geopedología, a dos escalas diferentes. Del análisis del paisaje derivaron la interpretación de relieves, cuya representación espacial tridimensional fue identificada, según las características externas y a través del análisis de su origen. La pedología correspondió al análisis de la génesis de suelos, en la cual, intervienen factores y procesos que pueden ser analizados, a través de la morfología y los inventarios taxonómicos. Los 3.238 perfiles de suelos analizados en la escala general 1:100.000 fueron un componente importante, en la identificación y en la delimitación de los ecosistemas colombianos actuales.

La información de unidades geopedológicas permitió definir un marco general con variantes, de acuerdo con la presencia de coberturas vegetales y el análisis de la biodiversidad. La similitud entre las delineaciones de geopedología y de ecosistemas muestra el aporte de la información de suelos en la delimitación de los ecosistemas terrestres, a las dos escalas representadas en Colombia.

En la cartografía de suelos, se contó con la descripción de las características orientadas al uso y manejo. En la representación de la geopedología, se identificaron los ambientes que configuran relieves, cuyo sustrato permitió la delimitación de ecosistemas, en el contexto nacional, a través del análisis del paisaje. En la delimitación de ecosistemas a la escala general, se analizó el marco evolutivo del suelo, por medio de los procesos de formación, como producto de la acción de las coberturas vegetales y los organismos, cuya intervención produjo sustratos diferentes. 
Los conceptos utilizados para generar el mapa de geopedología, se realizaron por agrupación de ambientes morfogenéticos, identificados en el nivel geomorfológico, como punto de encuentro, para el caso de la escala 1:500.000 y, a la escala 1:100.000, se analizaron los ambientes, en los cuales, se formaron los suelos y, para ello, se utilizó la base de datos de suelos del mapa integrado de suelos de Colombia.

A diferencia del enfoque productivo, el ambiental no tomó como elementos determinantes las fases cartográficas, que definen el manejo, salvo la erosión, para caracterizar los ecosistemas transformados. La geopedología, a escala 1:500.000, se concentra en los orígenes geomorfológicos o ambiente de formación de las geoformas y, a escala 1:100.000, la geopedología se inclinó a interpretar los ambientes de formación de suelos, utilizando como elementos cartografiables derivados de características fisicoquímicas de los perfiles de suelos. La sostenibilidad transita entre el enfoque productivo y el ambiental; la geopedología, como sustrato de ecosistemas, permitiría hacer buen uso del territorio.

Financiación. La investigación que originó este artículo fue financiada por los autores y el Centro de Investigaciones y Desarrollo Científico de la Universidad Distrital Francisco José de Caldas. Conflicto de intereses: Los autores declaran que no existen conflictos de intereses que arriesguen la validez de lo expresado en este artículo.

\section{BIBLIOGRAFÍA}

1. ABDELFATTAH, M.A.; PAIN, C. 2012. Unifying regional soil maps at different scales to generate a national soil map for the United Arab Emirates applying digital soil mapping techniques. J. Maps. 8(4):392-405. Disponible desde Internet en: http://www.tandfonline.com/doi/abs/10.1080/17445647.2012.746744 (con acceso 18/05/2016).

2. ABDELFATTAH, M.A. 2013. Pedogenesis, land management and soil classification in hyper-arid environments: results and implications from a case study in the United Arab Emirates. Soil Use and Managem. 29(2):279-294. Disponible desde Internet en: http:// onlinelibrary.wiley.com/doi/10.1111/sum.12031/ abstract (con acceso 18/05/2016).

3. ABER, J.; MELILLO, J. 2001. Terrestrial ecosytem. Harcourt Academic Press. Cengage Learning. (Estados Unidos). 556p.

4. ANDERSON, R.; ANDERSON, S. 2010. Geomorphology: The Mechanics and Chemistry of Landscapes.
Cambridge, University Press. (Estados Unidos). 637p.

5. BEGON, M.; TOWNSEND, C.; HARPER, J. 1996. Ecology from individual ecosystems. $4^{\text {ed }}$ - Blackwell Publishing. (Australia). 738p.

6. BIRKELAND, P. 1999. Soils and Geomorphology. Oxford University Press. (Estados Unidos). 430p.

7. BOHLEN, P.; HOUSE, G. 2009. Sustainable agroecosystem management. Integrating ecology, economics, and society. CRC PRESS. Taylor y Francis Group. Boca Raton, (Estados Unidos). 296p.

8. CLEMENTS, F.E. 1905. Research Methods in Ecology. University of Nebraska. Univ. Publ. Co., (Estados Unidos). 334p.

9. CORTÉS, A. 1974. Índice de productividad de los suelos para valoración catastral. Reunión Panamericana sobre los levantamientos integrados y el desarrollo de los países. Instituto Panamericano de Geografía e Historia. México. p.263-278.

10. DARWIN, CH. 2011. La formación del mantillo vegetal por la acción de las lombrices con observación de sus hábitos. Fecha del libro 1881. Biblioteca Darwiniana. CSIC. (España). 229p.

11. FAO. 1993a. El Estado Mundial de la Agricultura y la Alimentación. Las políticas de recursos hídricos y la agricultura. Colección FAO: Agricultura. (Italia). 26:8-18.

12. FAO. 1993b. Guidelines for land-use planning. FAO Development Serie 1. (Italia). 96p.

13. FITZPATRICK, E. 1971. Pedology: A Systematic Approach to Soil Science. Oliver y Boyd. (Estados Unidos). 306p.

14. GERRARD, A.J. 1981. Soils and landforms, an integration of geomorphology and pedology. Ed. George Allen \& Unwin (publishers) Itd. (Inglaterra). 219p.

15. GERRARD, A.J. 1992. Soils geomorphology and pedology. An integration of Pedology and Geomorphology. Ed. Melbourne : Chapman \& Hall. (Inglaterra). 269p.

16. GUTIERREZ, M. 2013. Geomorphology. CRC PRESS. Taylor y Francis Group. Boca Raton, London and New York. (Estados Unidos). 1001p. 
17. HUGGET, R. 2003. Fundamentals of Geomorphology. CRC PRESS. Taylor y Francis Group. Boca Raton, London and New York. (Estados Unidos). 458p.

18. IBÁÑEZ, J.; PÉREZ, R. 2015. Diversity of Soil-Landscape Relationships: State of the Art and Future Chalenges. En: Zinck, J.A.; Metternicht, G.; Bocco, G.; Del Valle, $\mathrm{H}$. (eds). Geopedology. An Integration of Geomorphology and Pedology for Soil and Landscape Studies. Springer. (Suiza). p.183-191.

19. IDEAM. 2001. El medio ambiente en Colombia. Los suelos: estabilidad. Productividad y degradación. (Colombia). p.228-277.

20. IDEAM, IGAC, IAVH, INVEMAR, SINCHI e IIAP. 2007. Ecosistemas continentales, costeros y marinos, Bogotá, D.C.: Imprenta Nacional de Colombia. + 1 CD-ROOM, 37 planchas.

21. IGAC. 1955. Estudio de Suelos Valle del Sibundoy. Mapa de suelos (material cartográfico) / INCORA, Bogotá, D. E. un mapa escala 1:20.000.

22. IGAC. 1964. Estudio detallado de los suelos del Valle Aluvial de río Zulia. Norte de Santander. (Colombia). $104 p$.

23. IGAC. 1968. Estudio semidetallado de suelos para catastro municipios San Zenón y San Sebastián Magdalena / Instituto Geográfico Agustín Codazzi, Sociedad Agrológica Colombiana. (Colombia). 228p.

24. IGAC. 1981. Pedología de la serie Tibaitatá y competidoras. Gloria de Benavides. Fraccionamiento y mineralización de nitrógeno en suelos volcánicos de la sabana de Bogotá. Esperanza Vargas de Rozo. (Colombia). 53p

25. IGAC. 1981. Metodología para la delimitación de áreas homogéneas de tierras. Centro Interamericano de Fotointerpretación (CIAF). (Colombia). 61p.

26. IGAC. 1982a. Mapa de suelos de Colombia escala 1:1.500.000. Memoria explicativa. Subdirección de agrología. Ed. IGAC. (Colombia). 86p.

27. IGAC. 1982b. Estudio general de suelos del municipio de Puerto Gaitán-Meta. 14 planchas Escala 1:100.000. Subdirección de agrología. Ed. IGAC. (Colombia). 214p.
28. IGAC. 1986. Mapa de clasificación de tierras por su capacidad de uso de la intendencia de Arauca. Bogotá, D.C. dos plancha cartográficas escala 1:250.000.

29. IGAC. 1989. Estudio detallado y semidetallado de suelos de los valles de los ríos Zulia y Pamplonita, Norte de Santander. 12 planchas Escala 1:25.000. Subdirección de agrología. Ed. IGAC. (Colombia). 216p.

30. IGAC. 1995. Conceptos básicos comunes a la pedología y geomorfología. Curso Intensivo de Geomorfología. Leonidas Mejía. Centro de Investigaciones en Percepción Remota (CIAF). (Colombia). 188p.

31. IGAC. 2007. Definición de Usos Alternativos y Sostenibles Para la Ocupación de las Tierras a Nivel Nacional. Subdirección de agrología. Ed. IGAC. (Colombia). 183 p.32-42 y $86-91$.

32. IGAC. 2013. Unidades geopedológicas del territorio colombiano. Subdirección de agrología. Ed. IGAC. (Colombia). 74p.

33. IGAC. 2015. Mapa digital de suelos de Colombia, escala 1:100.000. formato shapefile, versión en proceso de revisión. (Colombia).

34. IGAC. 2016. Suelos y Tierras de Colombia. Subdirección de Agrología. Bogotá, D.C. Tomo 1. 545 p. Tomo 2. $854 \mathrm{p}$.

35. INGEOMINAS. 1976. Mapa Geológico de Colombia. Escala 1:1'500.000. Disponible desde Internet en: http://www2.sgc.gov.co/Geologia/Mapas-historicos. aspx (con acceso 2/02/2016).

36. INSTITUTO GEOGRÁFICO MILITAR -IGM-. 1957. Estudio general de suelos del altiplano de Tunja - Sogamoso. (Colombia). 215p.

37. MALAGÓN, D. 1966. Apuntes pedológicos sobre un perfil de la serie Tibaitatá. Corporación Autónoma Regional de la Sabana de Bogotá y de los Valles de Ubaté y Chiquinquirá. (Colombia).

38. MALAGÓN, D. 1968. Algunos aspectos químicos y físicos de suelos en tres series se La Sabana de Bogotá. Corporación Autónoma Regional de la Sabana de Bogotá y de los Valles de Ubaté y Chiquinquirá. (Colombia).

39 MALAGÓN, D. 1969. Fraccionamiento del humus en las series más importantes de la Sabana de Bogotá y del 
Valle de Ubaté y Chiquinquirá. Agricultura Tropical. 15 (12).

40. MALAGÓN C., D. 1975. Mineralogía de suelos, sus relaciones con la génesis, fertilidad y clasificación de suelos. Subdirección Agrológica. Bogotá: IGAC. $689 p$.

41. MINAMBIENTE, IDEAM, INSTITUTO HUMBOLTD, INSTITUTO AMAZONICO SINCHI, INVEMAR, IIAP, PARQUES NACIONALES E IGAC. 2015. Mapa de ecosistemas continentales, costeros y marinos. (Colombia).

42. MINISTERIO DE AMBIENTE Y DESARROLLO SOSTENIBLE. 2013. Política para la gestión integral ambiental del suelo (GIAS). (Colombia). 138p.

43. MONTENEGRO, H.; MALAGÓN, D. 1990. Propiedades físicas de los suelos. Instituto Geográfico Agustín Codazzi, Subdirección Agrológica. (Colombia). 813p.

44. PELTIER, L. 1950. The geographic cycle in periglacial regions as it is related to climatic geomorphology. Ann. Assoc. Am. Geographers. (Estados Unidos). 40(3):214-236.

45. RAFFAELLI, D. 2010. Ecosystem Ecology: A New Synthesis. Cambridge University press. Cambridge; New York, Cambridge. University Press. (Estados Unidos). 172p.

46. RICO, A. 1931. Estudio agrológico del sistema nacional de riego número 6 San Carlos, Coahuila. Sec. Recur. Hidr. Depto. Agrol. (Méjico). Un mapa.

47. SARMIENTO, G. 1982. Los ecosistemas y la ecosfera. Editorial Blume, S.A. (España). 268p.

48. STODDART, D.R. 1968. Climatic geomorphology: Review and reassessment. Progress in Geography - Vol. l. (Londres). p.160-222.

49. TEIXEIRA, A.; BAPTISTA, S. 2000. Geomorfologia y Meio Ambiente. Ed. BCD. UNIAO DE EDITORES. S.A. (Brasil). 379p.

50. TOOMANIAN, N. 2013. Pedodiversity and Landforms. En: Ibáñez, J, and Bockehim, J. (eds). Ed. CRC Press Tylor and Francis Group. (Estados Unidos). p.133152.

51. TRICART, J. 1977. Ecodinámica. Ed. Supren. (Brasil). 97p.
52. USDA. 1992. Soil Survey Staff. Keys to Soil Taxonomy. United States Department of Agriculture. Soil Conservation Service, Fifth Edition. SMSS Technical monograph. (19):139-176. Disponible desde Internet en: http://www.nrcs.usda.gov/Internet/FSE_DOCUMENTS/nrcs142p2_052453.pdf (con acceso 17/05/2016).

53. VOLOBUEV, V. 1964. Ecology of Soils. Editorial: Israel Program for Scientific Translations, Jerusalem. (Israel). 260p.

54. WHITE, R. 2006. Principles and practice of soil science. Blackwell publishing. Fourth edition. (Australia). 363p.

55. WILD, A. 1993. Soils and environment. An introduction. Ed. Cambridge University press. (Cambridge-Inglaterra). 289p.

56. ZAPATA, R. 2006. Química de los procesos pedogenéticos. Facultad de ciencias. Universidad Nacional de Colombia. Sede Medellín. (Colombia). 358p.

57. ZINCK, J.A. 1970. Aplicación de la geomorfología al levantamiento de suelos en zonas aluviales. Barcelona, Venezuela: Ministerio de Obras Públicas. Ed. MOP. (Venezuela). 79p.

58. ZINCK, J.A. 1974. Definición del ambiente geomorfológico con fines de descripción de suelos. Cagua, Venezuela: Ministerio de Obras Públicas (MOP). Ed. MOP. (Venezuela). 114p.

59. ZINCK, J.A. 1977. Ensayo sistémico de organización del levantamiento de suelos. Maracay, Venezuela: Ministerio del Ambiente y de los Recursos Naturales Renovables. Ed. MARNR. (Venezuela). 189p.

60. ZINCK, J.A. 1980. Valles de Venezuela. El hombre y su ambiente. Petróleos de Caracas-Venezuela SA. Ed. Cuadernos Lagoven. (Venezuela). 150p.

61. ZINCK, J.A. 1987. Aplicación de la geomorfología de levantamientos de suelos en zonas aluviales y definición del ambiente geomorfológico con fines de descripción de suelos. Subdirección de Agrología. Ed. IGAC. (Colombia). 178p.

62. ZINCK, J.A. 1988. Physiography and soils. Ed. International Institute for Aerospace Survey and Earth Sciences - ITC. (Enschede, The Netherlands). 156p. 
63. ZINCK, J.A. 2013. Geopedology: Elements of geomorphology for soil and geohazard studies. Ed. ITC-Faculty of Geo-Information Science and Earth Obser-

Recibido: Abril 20 de 2016 vation. (Enschede, The Netherlands). 127p.

64. ZINCK, J.A.; METTERNICHT, G.; BOCCO, G.; DEL VALLE, H.F. 2016. Geopedology An Integration of Geomorphology and Pedology for Soil and Landscape Studies. Ed. Springer International Publishing. (Suiza). 548p.

Aceptado: Diciembre 16 de 2016

Cómo citar:

Castro Méndez, C.E.; Agualimpia Dualiby, Y. del C. 2017. Implementación del mapa de geopedología como base para la delimitación de unidades de ecosistemas a nivel nacional en Colombia. Rev. U.D.C.A Act. \& Div. Cient. 201(1): 175-185. 